\title{
Investigating the Impact of COVID-19 toward Depression Rate of Indonesian Senior High Schools Teachers
}

\author{
Helma Malini \\ Universitas Tanjungpura, Pontianak, Indonesia \\ helma.malini@untan.ac.id
}

\section{ARTICLE HISTORY}

$\begin{array}{ll}\text { Received } & : 2021-06-18 \\ \text { Revised } & : 2021-10-31 \\ \text { Accepted } & : 2021-11-04\end{array}$

\section{KEYWORDS}

Pandemic

COVID-19

Depression

Work Stress

Work Load

Role of Conflict

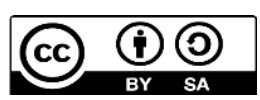

\begin{abstract}
The COVID -19 pandemic, which has been ongoing for over a year, has become a burden to the world, causing anxiety and depression. The purpose of this study is to investigate the depression and stress levels of senior high school teachers in Indonesia during the COVID-19 outbreak since they have experienced substantial teaching situations change as a result of it. In particular, the changes in their professional, as well as personal lives, pose challenges during the pandemic. This quantitative study distributed questionnaires to 50 high school teachers in Indonesia. The findings indicate that social support is the most significant factor $(0,46 \%)$ needed to prevent depression and stress among the teachers. Meanwhile, workload and role conflict cover $0,74 \%$ and $0,84 \%$ respectively of variables that teachers can change with the assumption that both variables exist even before a pandemic arises. This study also reveals that teachers continue to receive less attention and support, especially in terms of their stress and depression coverage. It is because the prevailing perception is that teachers should be the ones giving attention, not the ones receiving it. The study is that to withstand the Pandemic's uncertainty where high school teachers can minimize and overcome their depressions.
\end{abstract}

\section{Introduction}

The COVID 19 Pandemic affects human life, especially in education and social life. The Indonesian government determined to use online or distant learning methods to prevent COVID-19 spread in March 2020. The policy implementation is far from excellent, and the decision is made without much preparation (Iglesias-Pradas et al., 2021). The COVID-19 epidemic forced 165 countries to close schools, teachers must go above and above to keep nearly students earning. Teachers must always be creative, inventive, and enjoyable in delivering the information online learning through digital technology, so it has a favorable impact on students or students during the epidemic. COVID-19 (Bhumika, 2020).

Unsupported equipment and resources, in this case, the teacher, show inadequate preparation. Due to rapid policy changes, students suffer from sadness and stress. (Vladova et al., 2021). However, because of the imbalance of demands and abilities to cope with changes in the education sector caused by the COVID-19 pandemic, teachers may suffer from health issues and increased stress.
Concern parties such as parents and society highlight student stress and despair, but few recognize that teachers are equally affected (Bhumika, 2020; Realyvásquez-Vargas et al., 2020; Tan et al., 2020). Teachers have changed their teaching and learning methods, adjusted their learning plans, and dealt with uncertain situations as a result of the COVID 19 Pandemic. Teachers have changed their teaching and learning methods adjusted their learning plans and dealt with uncertain situations due to the COVID 19 Pandemic led to depressions (Tan et al., 2020).

When you are depressed, you experience emotions of melancholy and/or a loss of interest in activities that you previously enjoyed. It can result in several mental and physical problems, as well as a reduction in your capacity to operate at work and in your personal life. When it comes to depression in students, research produced during the COVID 19 pandemic in the realm of education continues to be focused on this issue, with various types of policies being implemented by various stakeholders to prevent student depression. Educators require a policy that is centered on them, one that pays attention to the problems of depression that they experience, and one that will ultimately assist them in facilitating teaching and learning activities throughout the pandemic (Baker, 2020; Evanoff et al., 2020; Iglesias-Pradas et 
al., 2021) Educational performance is, in essence, the level of performance demonstrated by instructors in the course of their professional responsibilities as educators. (Gopal et al., 2021; Kulikowski et al., 2021). According to this definition, performance is driven by processes, whether human or otherwise. According to the statement, performance can be classified as follows: work performance, work implementation, work accomplishment, work results, or work performance (Tan et al., 2020).

The quality of a teacher's performance will have a significant impact on the overall quality of educational outcomes. (Realyvásquez-Vargas et al., 2020), mostly since teachers are the individuals that have the most direct interaction with students during the learning process in educational institutions. When it comes to attempts to build human potential resources in the field of development, the teacher is fundamentally one of the components in the learning process that plays a (Lockee, 2021; Xiao et al., 2021). For a teacher to establish himself as a professional teacher, they must participate and position himself as a professional by the demands of a growing society. This requires integrity, loyalty, dedication, and responsibility on the part of the teacher, as well as on the part of the student.

As a result, the primary goal of this study was to examine how instructors have dealt with depression and to determine the amount to which they are affected by stress, anxiety, and depression. Although it is expected that teachers will be disproportionately affected by stress-related symptomatology, we also expect that the levels of anxiety, sadness, and stress will not be uniform across the sample. In other words, it is expected that there will be specific and significant differences between groups in the future. Our specific goal is to look into any variances based on gender, age, and amount of labor being done. In the future, it is expected that women would have higher levels of unfavorable psychological symptoms than men. It is also projected that younger teachers, those with a history of chronic illness, and those who have family members who suffer from chronic diseases may have higher levels of stress, anxiety, and despair.

Pandemic impositions that last for an extended period might be harmful. A hostile event that can result in severe mental health illnesses and depressions in teachers is a traumatic experience. It was discovered that quarantine was associated with high rates of sadness (31.2 percent) and anxiety (29.8 percent) even during the SARS pandemic (28.9 percent) (Tan et al., 2020). Similar to the 2009 H1N1 pandemic, substantial levels of anxiety were recorded during the outbreak; a study from China discovered that people in pandemic situations experience boredom, loneliness, irritation, worsening anxiety, and psychological distress (Gopal et al., 2021). Previous research, on the other hand, focused solely on one side of the equation, namely, pupils, and did not address the other side, which is the teachers, who are increasingly playing a vital role. As a result, the purpose of this study is to investigate how teachers have been affected by the pandemic in terms of their mental health and relationships in three major areas: job, family, and social interactions.

\section{Method}

This study is a survey study with a cross-sectional design that was analyzed using Data Analysis IBM SPSS version 22 software. In this study, 50 Senior High School Teachers from Indonesia took part in the survey that we select randomly by sending the questionnaire to schools. Online distribution of questionnaires is made possible by a google form. Work stress is the dependent variable in this study, which is determined by the independent variables, which are workload, role conflict, and social support. The research data were examined using univariate and bivariate employee status, as well as the frequency of occurrences depending on the independent and dependent variables, among other methods.

A descriptive statistical analysis is a tool to describe data. As defined by Widodo (2018), descriptive statistics are a data analysis technique that is used to describe the condition of research variables. This descriptive statistical test is used to provide an overview and description of data so that it can be easily understood by readers in the context of their research.

The information that has been gathered will be processed in stages to provide evidence in support of the hypothesis that has been put forward. The purpose of this study's multiple regression analysis is to determine the direction of the relationship between the independent variable and the dependent variable, whether each independent variable is positively or negatively related, and to predict the value of the dependent variable if the value of the independent variable increases or decreases over time.

The general formula for multiple linear regressions is as follows:

$$
Y=a+\beta 1 X_{1}+\beta 2 X_{2}+\beta 3 X_{3}+e
$$

\section{Testing of hypotheses}

A hypothesis is an assumption or a set of assumptions that may or may not be true. Hypotheses are frequently used as a basis for making decisions or as a reference for further research, among other things. In hypothesis testing, the acceptance or rejection of a hypothesis under investigation is the focus of the investigation. Parametric statistical testing, one of the statistical test tools, was used in this investigation. When the null hypothesis (H0) was determined, and 
the alternative hypothesis (Ha) was determined, parameter testing was carried out to evaluate the significance level.

\section{Test for the Coefficient of Determination (R2)}

The R2 test is used to determine how much the ability of the independent variable can explain the capacity of the dependent variable. Consequently, if the value of $\mathrm{R} 2$ is minimal, it indicates that the independent variable's ability to explain the dependent variable is limited. It is assumed that the independent variable can provide almost all the information required to predict the dependent variable.

The more accurate the regression model is with the existing data, and therefore, the more precise this model can be used to explain the dependent variable utilizing the independent variable, then it is assumed that it is close to one.

\section{Test of Statistical Significance (F Statistic)}

The F statistic test is designed to examine whether or not a given model is feasible, and it is used to determine whether or not the independent variables have a combined effect on the dependent variable. To put it another way, the $\mathrm{F}$ test determines whether all of the independent variables in the model have a combined effect on the dependent variable. Aside from that, the $\mathrm{F}$ test can be used to determine whether or not the regression model being employed is significant, given that the p-value is less than (0.05) and the $f$ count exceeds the $f$ table, which indicates that the model is significant and can be used to test the hypothesis. 0.05 is the degree of confidence for hypothesis testing when the level of confidence is 95 percent, or (5 percent).

\section{T. Statistical Evaluation}

The conclusion to determine this test is to determine whether or not there is a significant relationship between each independent variable and the dependent variable partially, specifically whether or not there is an influence between company size and profitability on purchasing decisions partially. Assuming that the t-count is statistically significant, which can be determined from the regression analysis, and that it is less than 5 percent, this indicates that the independent variable affects the dependent variable, as can be seen in the table above.

Bivariate analysis using Chi-Square $2 \times 2$ test with a significant level of $\mathrm{p}>0.05$ and $95 \%$ Confidence Interval. Multivariate analysis using Logistic Regression test where if the results show a significance value of $p<0.05$ means all independent variables as a whole are significant estimators for the dependent variable (Sugiyono, 2014).
Table 1. Respondent Demographics Profile

\begin{tabular}{lrr}
\hline \multicolumn{1}{c}{ Characteristics } & Number \\
\hline Age & $26-35$ & 15 \\
& $36-45$ & 18 \\
& $46-55$ & 17 \\
Gender & Female & 32 \\
& Male & 18 \\
Last Education & & \\
& Bachelor & 42 \\
& Master & 8 \\
Employment Status & & 14 \\
& Contract & 36 \\
\hline
\end{tabular}

Respondents aged 36-45 years were represented by 18 people; respondents aged 46-55 years represented by 17 people; and respondents aged 26-35 years represented by 15 people, as revealed by the results. Participants in this study included 32 women and 18 males who answered the survey's questions. With as many as 42 persons responding, Bachelor's degree is the most recent education they've received, while Strata 2 (S2) has as many as 8 people. The employee status of the respondents, who were divided into 36 civil servants and 14 contract workers, was determined.

\section{Result and Analysis}

Table 2. Distribution of respondents based on research variables

\begin{tabular}{rcc}
\hline \multicolumn{1}{c}{ Variable } & Number & Percentage \\
\hline Workload & & \\
High & 37 & 0,74 \\
Medium & 13 & 0,26 \\
Conflict Role & & 0,68 \\
High & 34 & 0,16 \\
Medium & 8 & \\
Social Support & & 0,36 \\
High & 18 & 0,64 \\
Medium & 32 & \\
Work Stress & & 0,84 \\
High & 42 & 0,16 \\
Medium & 8 &
\end{tabular}

The distribution of workload variables shows that 37 respondents $(0,74 \%)$ experienced high workload and 13 respondents $(0,26 \%)$ experienced low workload. While conflict role does exist with 34 respondents $(0,68 \%$ experienced high conflict role while 8 respondents $(0,16 \%)$ experienced low role conflict. Half of the total respondents received a high social support level, namely 18 respondents $(0,36 \%)$ and 32 respondents $(0,64 \%)$ accepted low social 
support. 42 respondents $(0,84 \%)$ experienced high work stress while low work stress was experienced by 8 respondents $(0,16 \%)$.

Respondents of this study reported having a significant amount of work to do, which included not only teaching but also guiding students, marking assignments, and correcting examinations. They also reported having a significant amount of administrative work outside of their job as teachers, as shown in the table above. These demands on instructors increase during times of pandemics or changes in the online teaching and learning system when teachers must develop new lesson plans while also adapting their teaching methods for use in online learning environments. While Junior High School and elementary school instructors utilize WhatsApp to facilitate online learning, senior high school teachers employ full online learning platforms such as Google Meet or Zoom to deliver their lessons to their students.

Meanwhile, 0.68 instructors at Senior High School, on the other hand, report experiencing substantial role tensions in their daily life. High school teachers in Indonesia must juggle the demands of the classroom, the demands of family life, and the demands of additional structural tasks associated with operating the school. Because of this role conflict, the effort increases, and there are no dividing lines between the numerous responsibilities during the epidemic and online learning sessions. Before online learning classes were mandated, there was a clear distinction between the teacher's job at school, at home, and in other responsibilities; but, once online learning classes were mandated, the line became one unit, increasing the level of sadness.

Teachers, on the other hand, have the smallest amount of social support available to them in comparison to the intense workload and conflict that they face as a result of the pandemic situation, in which everyone is caught up in their situation and often people only ask and pay attention to the most vulnerable party while paying less attention and focus to teachers, although teachers are also the most affected.

Table 3. The relationship between workload and work stress

\begin{tabular}{llll}
\hline \multicolumn{1}{c}{$\begin{array}{c}\text { Work } \\
\text { Load }\end{array}$} & \multicolumn{2}{c}{ Work Stress } & $p$-Value \\
& \multicolumn{1}{c}{ High } & \multicolumn{1}{c}{ Medium } & \\
\hline Medium & 35 & 4 & 0,000 \\
& $(74,5 \%)$ & $(13,8 \%)$ & \\
High & 12 & 25 & \\
& $(25,5 \%)$ & $(86,2 \%)$ & \\
N\% & $47(100 \%)$ & 29 & \\
& & $(100 \%)$ & \\
Total & $76(100 \%)$ & & \\
\hline
\end{tabular}

Table 3 revealed that the majority of teachers at the Senior High School suffered work stress as a result of the epidemic. A contributing factor was the transition from an offline to an online learning environment, as well as the adjustment from offline to online classrooms, which reduced the likelihood of receiving social support and spending time with friends.

According to (Novitasari et al., 2021), since the majority of Indonesian teachers also serve in administrative capacities, the relationship between workload and work stress is extremely significant. This might be perceived as most of the people overburdened with work, while the environment is changing as a result of the epidemic, and it required some time for everyone to get used to it. The increased workload associated with the preparation of learning materials that are packaged with interesting and easy to understand information that will later be displayed and taught to students when learning online using WhatsApp, Google Meet, or Zoom, which is very different from face-to-face learning systems, is experienced during online learning systems.

Following instruction, teachers conduct evaluations, verify collected student assignments, and generate reports, all of which add to the time required to complete the work. This condition is experienced by respondents in this study, and it has the potential to produce job stress. If this problem is not handled, it will have an adverse influence on the psychological and physical health of teachers.

In the event of a traumatic occurrence, teachers are at risk of experiencing psychological discomfort. The progression of the pandemic is unpredictable, and it may have long-term consequences for mental health. As a result, it is critical to establish a policy that includes the most effective interventions for identifying susceptible subgroups and planning for acute and long-term psychological services to regulate and reduce the burden of psychological disorders.

Table 4. The relationship between conflict role and job stress

\begin{tabular}{lccc}
\hline \multicolumn{1}{c}{$\begin{array}{c}\text { Conflict } \\
\text { Role }\end{array}$} & \multicolumn{2}{c}{ Work Stress } & $p$-Value \\
& High & Medium & \\
& & & \\
\hline Medium & 38 & $4(13,8 \%)$ & 0,000 \\
& $(80,9 \%)$ & & \\
High & $9(19,1 \%)$ & $25(86,2 \%)$ & \\
N\% & 47 & 29 & \\
& $(100 \%)$ & $(100 \%)$ & \\
Total & & \\
\end{tabular}


This result is in line with research conducted by (Gopal et al., 2021) on teachers in several high schools in India which shows that there is a relationship between the role of conflict with work stress where $33 \%$ of respondents with high role conflict experience work stress. According to Lockee, (2021), stress can cause severe depression that leads to mental health deterioration.

Meanwhile, a lack of awareness about the supporting technologies for teaching and learning in Indonesia's Senior High School is another aspect that contributes to role conflict. Most of the reason for this is the frequent change, which forces teachers to quickly adapt to the new environment. Meanwhile, regular work continues as usual, albeit with higher levels of stress as a result of the pandemic outbreak. When teachers are asked to explain and convey the changes that have occurred as a result of the pandemic to associated parties such as teachers and pupils, the role of conflict becomes even more important.

This research is in line with research conducted by (Chua, 2004). According to the report, teachers who are suffering role conflicts are more likely to experience increased work stress as a result of using online learning tools. Among the difficulties encountered are the dual jobs that must be performed at home, the restrictions of learning facilities such as inadequate technology, additional internet quota fees, and a poor internet network.

Table 5. The relationship between social support and job stress

\begin{tabular}{lccc}
\hline \multicolumn{1}{c}{$\begin{array}{c}\text { Conflict } \\
\text { Role }\end{array}$} & \multicolumn{2}{c}{ Work Stress } & $p$-Value \\
& High & Medium & \\
\hline Medium & $17(36,2 \%)$ & $21(72,4 \%)$ & 0,002 \\
High & $30(63,8 \%)$ & $8(27,6 \%)$ & \\
N\% & $47(100 \%)$ & $29(100)$ & \\
Total & \multicolumn{2}{c}{$76(100 \%)$} & \\
\hline
\end{tabular}

In this study, one of the primary objectives is to determine the association between social support and occupational stress. When it comes to dealing with pandemic circumstances, social support is becoming increasingly vital in the realm of human health. Social support comes from close relatives, friends, coworkers, neighbors, and pals in the teacher's surrounding area, as well as from online communities.

Teachers in high school benefit greatly from social support, which helps them maintain good mental health and reduce stress levels. In general, teachers receive social support from families, colleagues, and friends who live in the same or similar proximity to them in their professional lives. But when a pandemic strikes, teachers may have trouble gaining social support since the regularity with which they can meet with relatives, colleagues, and acquaintances is restricted by distance limitations and social distancing imposed by the government during times of emergency. Furthermore, social support is a type of moral assistance that should be delivered in the form of face-to-face interactions. However, because the majority of teaching activities are now conducted virtually, social support can only be acquired through the use of a computer monitor screen.

The results of the bivariate test in this study indicate that there is a relationship between social support with work stress $(\mathrm{p}=0.002)$. Research conducted by (Tan et al., 2020) in China in more than 7,000 respondents also indicated that there is a relationship between social support and work stress with a value of $\mathrm{p}=0.001$.

Based on current research, better levels of the social support offered by the surrounding environment are associated with decreased levels of stress and depression experienced by high school teachers. Teachers, on the other hand, are frequently adults who are expected to contribute rather than receive help, and as a result of this social shame, the lack of social support provided to teachers, both ethically and in terms of attentive care, is magnified.

It appears from the findings of this study that instructors do not receive the ideal degree of social support since they are experiencing conflict in their roles as educators and administrators. Teachers must pay attention to their kids while on the job, and they must also pay attention to their families when they return home from school. As a result of this conflict in roles, the social assistance supplied to teachers is not at its best.

Table 6. Logistic regression analysis of job stress

\begin{tabular}{|c|c|c|c|c|c|}
\hline \multirow[t]{2}{*}{ Variable } & \multirow[t]{2}{*}{ B } & \multirow{2}{*}{$\begin{array}{l}\text { P- } \\
\text { Valu } \\
\text { e }\end{array}$} & \multirow[t]{2}{*}{ OR } & \multicolumn{2}{|c|}{$95 \% \mathrm{CI}$} \\
\hline & & & & $\begin{array}{l}\text { Lowe } \\
\mathrm{r}\end{array}$ & Upper \\
\hline Work & - & 0,001 & 0,013 & 0,001 & 0,157 \\
\hline Load & 4,361 & & & & \\
\hline Role & - & 0,000 & 0,012 & 0,001 & 0,131 \\
\hline Conflict & 4,447 & & & & \\
\hline $\begin{array}{l}\text { Social } \\
\text { Support }\end{array}$ & 3,235 & 0,002 & 25,402 & 2,408 & 267,987 \\
\hline
\end{tabular}

Meanwhile, using logistic regression, Table 6 attempts to demonstrate which variable has the most influence on job stress. Table 6: According to the findings of the study, high school teachers experience increased job stress as a result of a lack of social support from their colleagues. When compared to workload variables and work conflicts, a low degree of social support has a p-value of 0.002 and an odds ratio of 25.402, indicating that it is 25 times more 
likely to cause work stress. Therefore, teachers can make adjustments to their workload, and even when the workload increases during the pandemic, teachers can make adjustments to account for the increased effort. Meanwhile, due to the separation of roles at work as a teacher with additional administrative responsibilities and the role of teachers at home, the role conflict can be adjusted by instructors so that it does not generate stress at the workplace.

Furthermore, the purpose of this study is to establish which variables have the most impact on the occurrence of work stress. Following the results of the logistic regression test, it was discovered that social support is the variable most related to job stress $(\mathrm{p}=$ 0.002 ), with an odds ratio of 25.402 for this variable. Low social support received by respondents was found to be 25.4 times more likely to cause work stress.

Social support can help people become more mentally resilient, especially when faced with the uncertainty that we all face. Teachers are concerned about when the situation will return to normal, as well as the possibility of health issues resulting from the Covid-19 pandemic, which they are experiencing. Teachers should get support from all relevant parties, which in this case includes not only the government but also the surrounding environment, particularly from the parents of pupils who are enrolled in the school. Teacher forums and communities can also be a source of social support for one another, particularly when it comes to providing answers to difficulties that arise during a pandemic.

The unsettled nature of educational environments, as well as the life stage of teachers who are "in transition" from face-to-face to online learning during a sensitive career shift, renders them particularly vulnerable to the negative psychological effects of traumatic experiences. The psychological distress levels measured in our study are consistent with this interpretation: students' concerns about their teaching activities, both in terms of delays regarding the completion of the pandemic and their sense of loneliness and isolation due to physical distance from their peers and partners concerning COVID-19 effects and containment measures, are associated with the psychological distress levels measured in our study.

Research by (Marazziti et al., 2021; Tan et al., 2020) stated that when someone is experiencing a life crisis, social support becomes extremely crucial, according to the author of the book. To avoid being corroded by despair and stress, social support becomes a pillar of strength. According to the findings of the study, 85 percent of instructors who have complete social support may also perform effectively. Meanwhile, according to another study, teachers who do not receive adequate social support have poor teaching effectiveness and do not achieve the desired results (Lockee, 2021).

\section{Discussion}

During the COVID-19 conference in Indonesia, this study examined the level of depression among senior high school teachers. According to the findings, teachers are not only overwhelmed by the epidemic that has occurred, but they are also experiencing feelings of concern as a result of changes in learning methods and a lack of social support available to them in their profession. Generally speaking, teachers conceal and hide their feelings from the wider public, and the recent pandemic may have exacerbated this problem even further. Teachers, according to studies, endure uncertainty during a pandemic, which results in stress and anxiety for them. Teachers may have fewer coping methods available to them during times of uncertainty and stress. Teachers in this study, notably those in the age range of 36 to 45 years, reported higher levels of anxiety as compared to their older counterparts. As is well known, younger teachers are continuously burdened with more work than they can handle, which can lead to an increase in despair. Although work overload provides an opportunity to obtain job experience. During a pandemic, on the other hand, the experience is restricted to screens only.

Teachers in senior high school appeared to have a higher degree of worry than their students, which led to the hypothesis that teachers in Junior high school and Primary high school suffer a higher level of anxiety during times of epidemics or pandemics, according to the findings from (Hawes et al., 2021; Tan et al., 2020), there is no definitive explanation has been provided, although it's possible that junior and primary school teachers were more informed about what to expect as the pandemic progressed than the senior high school teacher.

The highest levels of anxiety were experienced by teachers who were staying alone, as opposed to those who were staying with family and friends. In the current situation, those who are remaining alone are typically separated from their families, and the sudden threat to their safety and security during this epidemic could have made these students feel even more isolated while posing issues from various perspectives. Tracking long-term loneliness and intervening quickly is critical in reducing feelings of anxiety because they promote a sense of belonging and help people feel less isolated. Mental and social well-being must establish and sustain relationships, which is one of the features of a teacher's life. Unfortunately, the COVID19 pandemic has triggered a "social recession"-a pattern of social distancing that has persisted beyond the immediate pandemic and is resulting in a lack of emotional support as well as broader societal consequences, such as elevated levels of anxiety among the general public.

The most often mentioned stressors were mostly financial limits, remote online classes, and worry 
about the future as a result of COVID-19 and lockdowns, according to the survey. In terms of finances, the students were concerned about their ability to manage their school financial obligations as a result of their family's loss of income as well as their loss of opportunity to work and self-finance their education. It is particularly shocking to see that some Teachers were required to attend $6-8$ hours of daily online classes, which added to their already overwhelming stress and health problems. The fact that they were uncertain about the situation had a significant impact on their anxiety levels. Teachers, particularly those who bear an additional burden of work, have reported feeling a great deal of stress and anxiety as a result of being overburdened with ongoing alternative evaluations.

This research also revealed that teachers who taught online classes experienced higher levels of anxiety. This could be related to the prominence of the physical surroundings, and the fact that they are more familiar with health issues than the general public puts them at greater risk of psychological distress. Furthermore, this finding could be explained by the fact that instructors who are more invested in their professions are normally more empathic and altruistic, and as a result, they are more likely to experience negative psychological reactions in a health crisis. Moreover, this result is consistent with prior studies, which found that those living near the area's most hit by the pandemic experienced higher levels of psychological distress (in our case, in Pontianak).

Teachers were more likely than the general population to experience anxiousness. This discovery is similar to the one made by (Alzueta et al., 2021; Bhumika, 2020), when compared to other professions, teachers do not express their feelings as frequently, and the recent epidemic may have aggravated the problem. The uncertainty tolerance threshold of teachers is lower than that of other professions and passing that threshold causes undue tension and worry in the classroom. Teachers may have fewer coping methods available to them in times of uncertainty and stressful conditions.

Our findings also indicate that increasing the willingness of teachers to assist in efforts to contain the pandemic was associated with increased levels of worry, indicating that the experience of losing control over one's health risk management can cause psychological suffering. According to the findings of this study, there is a relationship between teacher and anxiety level, which is consistent with earlier studies that have found that females are more likely than males to have anxiety symptoms in response to health emergencies and imposed quarantines.

It is advised that additional studies should be conducted to incorporate proven coping tactics employed by teachers during testing times, such as the
COVID-19 epidemic. Students' anxiety and adverse psychological effects should be minimized through the use of effective teaching, learning, and assessment methods in this new normal environment. This can have the dual benefit of increasing learning outcomes while also reducing anxiety and adverse psychological impacts. To conclude, more research should be conducted into the utilization of digital technology and psychological artificial intelligence solutions to manage the anxiety levels of instructors.

\section{Conclusions}

Teachers are not only overwhelmed by the epidemic that has occurred, but they are also experiencing feelings of concern as a result of changes in learning methods and a lack of social support available to them. It was discovered from the demographic profile of high school teachers who participated in this study that instructors with Civil Servant status possessed greater mental resilience than teachers employed as independent contractors. This mental fortitude is attributed to the certainty of having a job during a pandemic, which allows them to remain more focused on their jobs even when faced with the increased workload, the role of conflict, and a lack of social support from their colleagues. To the best of our knowledge, this is the first study to evaluate the influence of the COVID-19 epidemic on the mental health and psychological well-being of teachers. Studies on this topic are still in their infancy. The sample and cross-sectional nature of our study, which limits causal interpretations, are the most significant limitations of our study. The use of selfreported measures rather than clinical diagnoses of anxiety and depression was a further drawback, even if the scales used were validated and were widely used in the field of psychology. The majority of instructors are experiencing depression-related difficulties such as anxiety, depression, and trauma as a result of Covid-19. Some of the most significant risk factors include social alienation and isolation, economic crisis, stress, and traumatic experiences. It is recommended that the government give services to assist instructors in dealing with the psychological harm that Covid-19 poses to them as a result of this problem.

As a result, in the Pandemic's uncertainty, related parties, in this case, the government, must direct policies toward teachers, particularly by providing psychological counseling services and providing training or seminar facilities, so that teachers can survive the uncertainty of the pandemic. It is advised that additional studies be conducted to incorporate proven coping tactics employed by teachers during testing times, such as the COVID-19 epidemic. Research should also be directed on teaching, learning, and assessment approaches in the "new normal" domain, which can have the dual benefit of increasing student learning outcomes while simultaneously 
reducing teacher anxiety and detrimental psychological effects on their students and colleagues. Finally, more attention should be paid to studies into the usage of digital technology solutions to reduce the anxiety levels of teachers.

\section{References}

Alzueta, E., Perrin, P., Baker, F. C., Caffarra, S., Ramos-Usuga, D., Yuksel, D., \& Arango-Lasprilla, J. C. (2021). How the COVID-19 pandemic has changed our lives: A study of psychological correlates across 59 countries. Journal of Clinical Psychology, 77(3), 556-570. https://doi.org/10.1002/jclp.23082

Baker, M. G. (2020)., Who cannot work from home? Characterizing occupations facing increased risk during the COVID-19 pandemic using 2018 BLS data. Medrxiv.

Bhumika. (2020). Challenges for work-life balance during COVID-19 induced nationwide lockdown: exploring gender difference in emotional exhaustion in the Indian setting. Gender in Management, 35(7-8), 705-718. https://doi.org/10.1108/GM-06-2020-0163

Chua, B. S. (2004). Stres Pekerjaan, Kepuasan Kerja, Masalah Kesihatan Mental dan Strategi Daya Tindak: Satu Kajian di Kalangan Guru Sekolah di Kota Kinabalu, Sabah. Jurnal Teknologi, 40(1), 1- 18. https://doi.org/10.11113/jt.v40.426

Evanoff, B. A., Strickland, J. R., Dale, A. M., Hayibor, L., Page, E., Duncan, J. G., Kannampallil, T., \& Gray, D. L. (2020). Work-related and personal factors associated with mental well-being during the COVID-19 response: survey of health care and other workers. Journal of Medical Internet Research, 22(8), e21366. https://doi:10.2196/21366

Gopal, R., Singh, V., \& Aggarwal, A. (2021). Impact of online classes on the satisfaction and performance of students during the pandemic period of COVID 19. Education and Information Technologies, 1-25. https://doi: 10.1007/s10639021-10523-1.

Hawes, M. T., Szenczy, A. K., Klein, D. N., Hajcak, G., \& Nelson, B. D. (2021). Increases in depression and anxiety symptoms in adolescents and young adults during the COVID-19 pandemic. Psychological Medicine, 13, 1-9. https://doi.org/10.1002/jclp.23082

Iglesias-Pradas, S., Hernández-García, Á., ChaparroPeláez, J., \& Prieto, J. L. (2021). Emergency remote teaching and students' academic performance in higher education during the COVID-19 pandemic: A case study. Computers in Human Behavior, 119, 106713. https://doi.org/ 10.31014/aior.1993.04.02.303
Kulikowski, K., Przytuła, S., \& Sułkowski, L. (2021). E-learning? Never again! On the unintended consequences of COVID-19 forced e-learning on academic teacher motivational job characteristics. Higher Education Quarterly. 2021;00:1-16. https://doi.org/10.1111/hequ.12314

Lockee, B. B. (2021). Shifting digital, shifting context: considering teacher professional development for online and blended learning in the COVID-19 era. Educational Technology Research and Development, 69(1), 17-20. https://doi.org/10.1007/s11423-020-09836-8

Marazziti, D., Cianconi, P., Mucci, F., Foresi, L., Chiarantini, C., \& Della Vecchia, A. (2021). Climate change, environment pollution, Covid19 pandemic and mental health. Science of The Total Environment, 773, 145-182. https://doi.org/10.1016/j.scitotenv.2021.145182

Novitasari, D., Asbari, M., Purwanto, A., Fahmalatif, F., Sudargini, Y., Hidayati, L. H., \& Wiratama, J. (2021). The Influence of Social Support Factors on Performance: A Case Study of Elementary School Teachers. International Journal of Social and Management Studies, 2(1), 41-52. https://doi.org/10.5555/ijosmas.v2i1.6

Realyvásquez-Vargas, A., Maldonado-Macías, A. A., Arredondo-Soto, K. C., Baez-Lopez, Y., Carrillo-Gutiérrez, T., \& Hernández-Escobedo, G. (2020). The impact of environmental factors on academic performance of university students taking online classes during the COVID-19 Pandemic in Mexico. Sustainability, 12(21), 122. https://doi.org/10.3390/su12219194

Reinke, W. M., Stormont, M., Herman, K. C., Puri, R., \& Goel, N. (2011). Supporting children's mental health in schools: Teacher perceptions of needs, roles, and barriers. School Psychology Quarterly,26(1), 1-13, https://doi.org/10.1177\%2F1098300712459079

Sugiyono. (2014). Metode Penelitian Kuantitati Kualitatif Dan R\&D. CV. Alfabeta.

Tan, W., Hao, F., McIntyre, R. S., Jiang, L., Jiang, X., Zhang, L., Zhao, X., Zou, Y., Hu, Y., \& Luo, X. (2020). Is returning to work during the COVID19 pandemic stressful? A study on immediate mental health status and psychoneuroimmunity prevention measures of Chinese workforce. Brain, Behavior, and Immunity, 87, 84-92. doi: 10.1016/j.bbi.2020.04.055

Traunmüller, C., Stefitz, R., Gaisbachgrabner, K., \& Schwerdtfeger, A. (2020). Psychological correlates of COVID-19 pandemic in the Austrian population. BMC Public Health, 20(1), $1-16$. https://doi.org/10.1080/13548506.2021.1955137 
Vladova, G., Ullrich, A., Bender, B., \& Gronau, N. (2021). Students' Acceptance of TechnologyMediated Teaching-How It Was Influenced During the COVID-19 Pandemic in 2020: A Study From Germany. Frontiers in Psychology, 12 ,

(69),

$1-15$. https://dx.doi.org/10.3389\%2Ffpsyg.2021.63608 6

Xiao, Y., Becerik-Gerber, B., Lucas, G., \& Roll, S. C. (2021). Impacts of working from home during COVID-19 pandemic on physical and mental well-being of office workstation users. Journal of Occupational and Environmental Medicine, 63(3):

181-190.

https://doi.org/10.1002/jclp.23082 